\title{
Breast implant-associated anaplastic large cell lymphoma vs. anaplastic large cell lymphoma in a patient with breast implant effusion history: a diagnostic dilemma
}

\author{
Alexandros Iliadis $^{1}$ (D) $\cdot$ Triantafyllia Koletsa $^{1} \cdot$ Alkinoos Athanasiou $^{2} \cdot$ Niki Stavroyianni $^{3}$. \\ Athanasios Spiliotopoulos ${ }^{2} \cdot$ Maria Papaioannou $^{4}$ - Ioannis Kostopoulos ${ }^{1}$
}

Received: 2 December 2016 / Accepted: 30 January 2017 /Published online: 9 February 2017

(C) Springer-Verlag Berlin Heidelberg 2017

\begin{abstract}
Breast implant-associated anaplastic large cell lymphoma is a rare tumor, affecting patients in the post-breast implant setting, first reported in 1997. We report an extraordinary case of anaplastic large cell lymphoma presenting not as a breast mass but as a spinal tumor, and thus in distant disease stage if considered breast implant-associated. A 35-year-old female, who had undergone bilateral breast augmentations over a decade ago and recent implant removal, complained of back pain with an anterior thoracic projection bilaterally. Clinical and radiological work-up revealed pleural nodules, T6 vertebral/costal infiltration, enlarged left axillary lymph nodes, and extradural spinal masses bilaterally, from which tumor samples were studied morphologically and immunohistochemically. There was diffuse infiltration by enlarged, neoplastic lymphoid cells with eosinophilic cytoplasm and large, irregular, partially embryoid, or multi-lobulated nuclei with distinctive nucleoli. The findings were interpreted as soft tissue and bone infiltration by an aggressive $\mathrm{CD} 30^{+}$, $\mathrm{ALK}^{-}$anaplastic large cell lymphoma. Since cytological or histological analysis on the initial peri-prosthetic
\end{abstract}

Triantafyllia Koletsa

koletsa@med.auth.gr

1 Department of Pathology, Faculty of Medicine, Aristotle University of Thessaloniki, 54124 Thessaloniki, Greece

2 1st Department of Neurosurgery, AHEPA University Hospital, Faculty of Medicine, Aristotle University of Thessaloniki, 541 24 Thessaloniki, Greece

3 Hematology Department and BMT Unit, 'George Papanicolaou' Hospital, 57010 Thessaloniki, Greece

$4 \quad$ 1st Department of Internal Medicine, Hematology Unit, AHEPA University Hospital, Faculty of Medicine, Aristotle University of Thessaloniki, 54124 Thessaloniki, Greece inflammation tissue and effusion was not performed, the diagnosis of breast implant-associated anaplastic large cell lymphoma is critically difficult. If the clinical setting is not regarded coincidental, then it strongly favors the diagnosis of what could be a rare aggressive undetected form of breast implant-associated anaplastic large cell lymphoma. If so, the importance of awareness of this probable clinical entity among female patients and their surgeons as well as of the recognition and cytological study of peri-prosthetic effusions is emphasized, so as to enable early detection of a possible lymphomatous proliferation.

Keywords Peri-prosthetic effusion $\cdot$ Post-implantectomy setting $\cdot$ Extradural spinal cord mass $\cdot$ ALK-negative anaplastic large cell lymphoma $\cdot$ Hematopoietic T cell neoplasm

\section{Introduction}

Breast implant-associated anaplastic large cell lymphoma (BIA-ALCL) is a rare tumor with an incidence of $0.3 \%$ per 100,000 women per year and an average patient age of 50 years, affecting patients in the post-breast implant setting. It was first reported in 1997 [1], 35 years after insertion of the first silicone breast implant. The US Food and Drug Administration reported 60 documented cases in 2011 with the finding that breast implants increase the risk by an estimated odds ratio of 18.2 , and an absolute risk ranging at $1 / 500,000-3,000,000$ women with implants per year [2]. Recent reviews of published cases showed interesting clinical findings: median time to diagnosis after implantation of approximately 10 years, most common type of presentation as a peri-implant effusion and a less common one as an adjacent 
mass to the effusion or a peri-prosthetic capsular contracture additionally [3, 4].

BIA-ALCL is thought to be an atypical lymphoid proliferation that lies at the interface between benign and malignant, a cytologically alarming lesion that is selflimited if anatomically confined to the seroma cavity [5]. Provided there is a presence of a capsule surrounding the implant and confining the proliferation, the clinical behavior seems to be benign. Progression risk increases, when the neoplastic cells escape this compartment. It is possible that chronic inflammatory stimulation may be related to the development of BIA-ALCL, given the immunogenic potential of implant materials, the newly detected bacterial biofilm [6] and the fact that ALCL originates from activated mature cytotoxic $\mathrm{T}$ cells [7]. Thus, foreign biomaterial may incite neoplastic transformation, probably by instigating a chronic inflammatory response. Although BIA-ALCL resembles anaplastic lymphoma kinase-1 negative $\left(\mathrm{ALK}^{-}\right)$primary cutaneous ALCL with indolent clinical course, many unique clinical and pathological features suggest that it is a separate lymphoproliferative entity [8], a novel manifestation of site- and material-specific lymphoma, demonstrating a wide array of diverse characteristics [2].

\section{Clinical history}

We recently encountered in routine practice a case of a 35year-old female, who had previously undergone bilateral breast augmentations almost 12 years ago. Implant material was silicone. A small peri-prosthetic recurrent isolated effusion in the post-implantation period was first reported nearly 2 years before, not resulting in fluid aspiration and cytological analysis, in the absence of other classical presenting clinical symptoms such as peri-implant pain/discomfort, contracture, breast asymmetry, radiographic evidence of capsular fibrosis, or mass. After about 1 year, the implants were removed due to a local inflammation, without implant replacement. No histologic evaluation was recorded. Six months later, the patient complained of back pain with an anterior thoracic (radicular T6) projection bilaterally. Clinical and radiological work-up revealed pleural effusions with pleural nodules (Fig. 1a), enlarged left axillary lymph nodes, mass and infiltration of vertebra and ribs at $\mathrm{T} 6$ level, and extradural spinal tumor at $\mathrm{T} 5$ to T7 levels (Fig. 1b, c). These findings raised suspicion of a neuropathological neoplasia and were neurosurgically managed. T6 and partial T5 and T7 laminectomy was performed to allow for the epidural mass excision and a bilateral decompression of T6 roots. The postoperative period was uneventful; the patient's symptoms were alleviated, and she was discharged at the fifth post-op day.
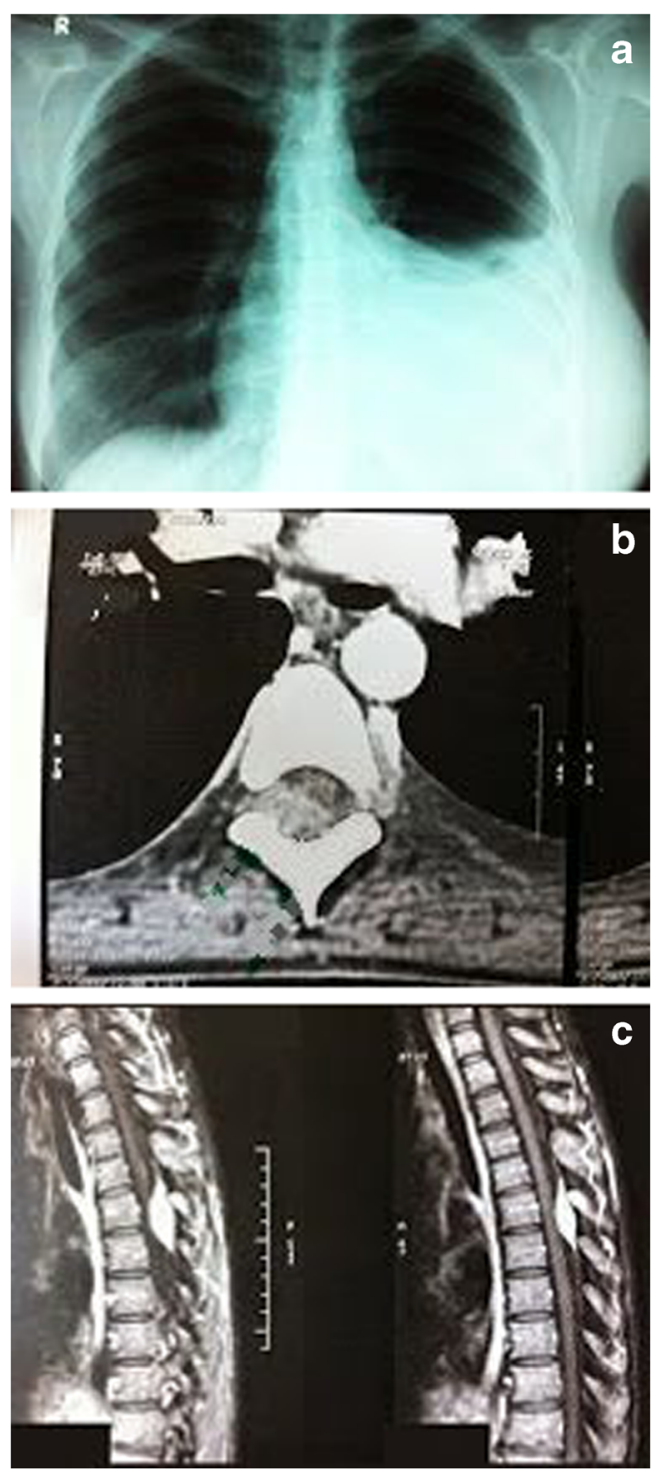

Fig. 1 Radiologic imaging of thorax: pleural effusion on plain chest $\mathrm{X}$ ray (a), enhancing extradural spinal mass on T6 level axial CT with contrast medium (b), and sagittal T1-weighted MRI with fat saturation and gadolinium enhancement (c)

\section{Materials and methods}

A few tumor samples were sent intraoperatively for frozen sections and more specimens followed later, including bone tissue. It was all fixed in $10 \%$ formalin buffer, demineralized where needed, embedded in paraffin blocks, and stained with hematoxyline/eosine. Serially cut sections were studied morphologically and immunohistochemically.

\section{Results}

Most tissue samples showed diffuse infiltration by enlarged, neoplastic lymphoid cells with eosinophilic cytoplasm and 
large, irregular, partially embryoid, or multi-lobulated nuclei with distinctive nucleoli (Fig. 2a), some of which showing eccentric "horseshoe-" or "kidney-shaped" nuclei with a paranuclear eosinophilic region, identified as "hallmark" cells (Fig. 2b). Binuclear or multi-nuclear aberrant cell forms were also to be seen, as well as smaller cells containing the aforementioned nuclei. Mitotic rate was increased with the presence of atypical tripolar mitoses. Neoplastic cell masses were also found within the intertrabecular medullary spaces in the bone tissue samples. The following immunophenotype was obtained: CD30+ (Fig. 2c), CD45+, EMA+, CD43+ (Fig. 2d), CD15+, CD2+ (Fig. 2e), CD4+, granzymeB+ (Fig. 2f), perforin,+ CD45RO-, CD3-, CD5-, CD7-, CD8 -, CD56-, TIA1-, ALK1-, LMP1-, CD20-, CD45RA-, PAX5-, CD79a-, and AE1/AE3-. The findings were interpreted as soft tissue and bone infiltration by an aggressive CD30+, ALK-, and ALCL.
Staging procedures showed stage $\mathrm{IV}_{\mathrm{A}}$ with an age-adjusted International Prognostic Index (aaIPI) of 1, falling in the lowintermediate risk category. The patient was treated with $6 \mathrm{cy}$ cles of chemotherapy with cyclophosphamide, hydroxydaunorubicin, oncovin, etoposide, and prednisone (CHOEP) in combination with central nervous system (CNS) prophylaxis and achieved complete response. Consolidation therapy with autologous hematopoietic stem cell transplantation (HSCT) followed, and the patient is still in complete remission, after 3 years of follow-up.

\section{Discussion}

In the context described above, we would like to report an extraordinary case of a probable BIA-ALCL presenting not as a breast mass, but as a spinal tumor, and thus if so in distant
Fig. 2 Morphological and immunophenotypical features: predominant population of large cells with irregular nuclei (a). Note large hallmark cells showing eccentric horseshoe- or kidneyshaped nucleus and abundant eosinophilic cytoplasm (b). All malignant cells are strongly positive for $\mathrm{CD} 30 / \mathrm{Ki}-1$ (c), T cell markers CD43 (d) and CD2 (e), and cytotoxic marker granzymeB (f) $(a-b \mathrm{HE} \times 400, c-f$ IHC $\times 200)$
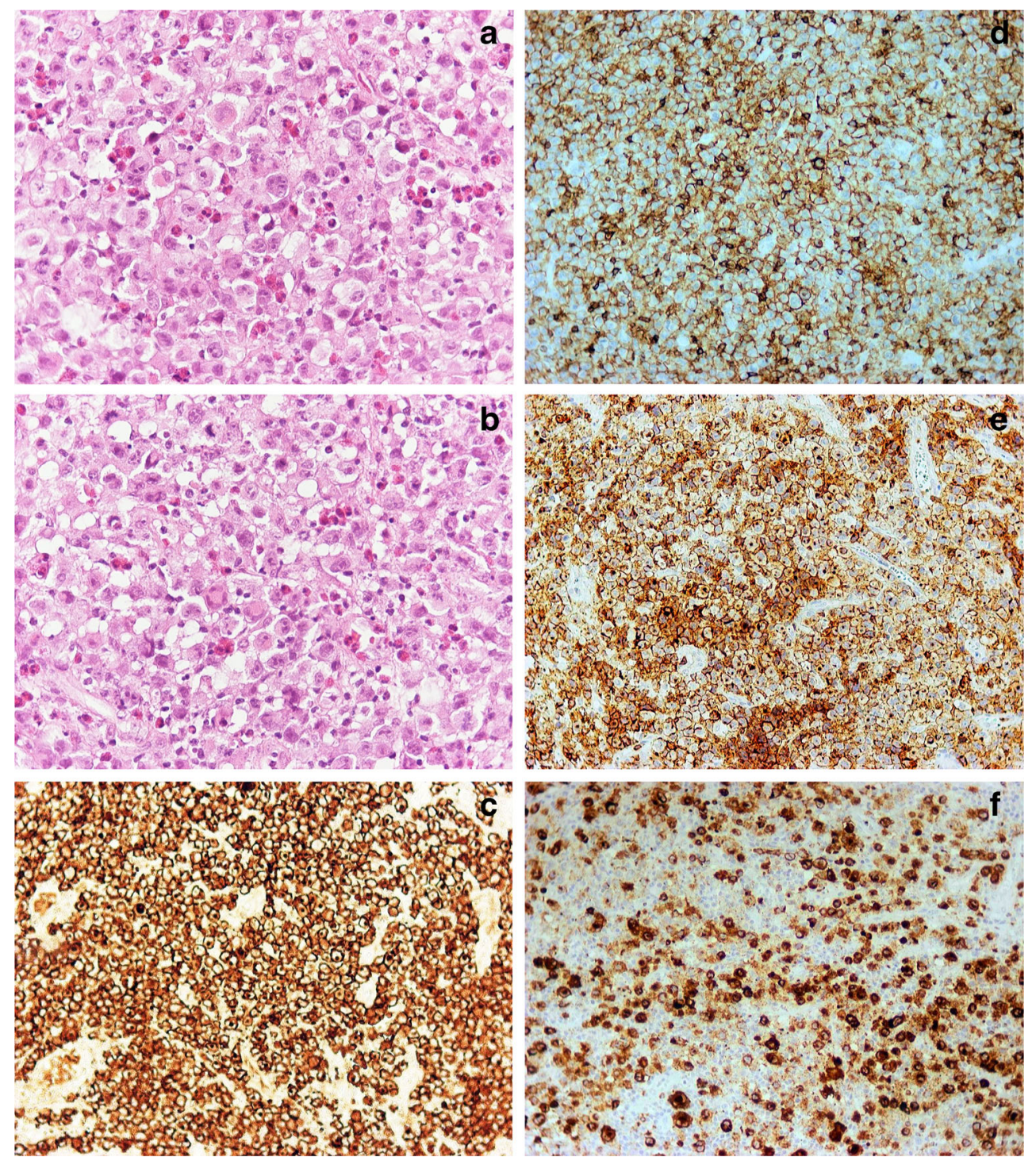
disease stage. Unique features of this case include young age of presentation and aggressive biological behavior possibly initiated by a late-onset recurrent limited effusion, evolving to local inflammation and rapidly resulting in a postresection generalized neoplastic involvement, following a pleurocostal and vertebrospinal dissemination path on the postero-mammary transverse plane, as well as regional node metastasis. Delayed detection, non-compliant patient, inadequate clinical surveillance, and delayed referral for surgical intervention may have well contributed to the striking and brief disease course from isolated effusion to epidural mass.

The presence of any sort of effusion beyond 1 year after implant surgery is relatively rare, estimated at less than $1 \%$, and any "late-onset seroma" formation should raise the suspicion of BIA-ALCL [9]. Any patient presenting with recurrent seroma 6 months or more after implantation should undergo aspirate analysis without delay [10]. The lack of routine pathology examination of seroma or excised tissue could result in an underestimation of ALCL occurrence [3]. The real incidence of BIA-ALCL may be higher, as it is known that seroma effusions are not being consistently submitted for microscopic examination, as in this case. Often swollen breasts are assumed to be infected. Implant-associated neoplasms appear to be difficult to identify at imaging, and patients with a mass have a worse progression-free survival than those without [11]. There is a need to better understand the imaging findings in patients with BIA-ALCL, particularly in regards to the detection of an effusion or an implant-related mass. Because this neoplasm can be subtle and easily overlooked, it is important to carefully examine the gross specimen removed for implantrelated complications [7]. However, in cases where a thin and discontinuous tumor cell layer along the inner surface of the capsule is the only finding, histopathological examination can be challenging and requires extensive sampling.

After many reports directed attention to the need of definition of BIA-ALCL as a distinct clinicopathological entity [12], according to the 2016 upcoming revised World Health Organization (WHO) classification of hematological malignancies, BIA-ALCL has been designated as a new provisional entity, separated from other $\mathrm{ALK}^{-}$ALCL and associated with very good prognosis; however, its non-invasive character is not absolute and is lost with invasion of the capsule [13]. Thus, if the correlation with the breast implant is acknowledged, the aggressive clinical course of this case categorizes it as the "infiltrative" BIA-ALCL clinicopathological variant, even though undetected and albeit without an initial mass, the other being the "in situ" variant [14]. While the total capsulectomy is the conservative treatment of choice for optimal event-free survival [15], this may not have been the case here taking into account the unavailable data. Because microscopic proof of neoplastic cell presence in the effusion or neoplastic tissue film attachment to the capsule cannot be provided, the question on the relationship between the development of this disseminated
$\mathrm{CD}^{+} 0^{+} \mathrm{ALK}^{-} \mathrm{ALCL}$ and the removed breast implant cannot be answered with the highest degree of assurance. The identical/ similar features of the entire clinical setting (e.g., age, implantation-to-diagnosis interval, topographical spread pattern) as described in the existing case reviews, logically minimize the possibility of an unrelated coincidental de novo extradural thoracic ALCL and may render the relationship as nothing else but causative. The factors contributing to progression have not been demarcated [13].

A small subset of cases has been reported to be aggressive with several well-documented fatal cases [8]. The aggressive clinical course of some BIA-ALCL may be due to intrinsic behavior of the initial tumor clone or may represent an advanced stage of disease at diagnosis with already established distant micrometastases [16]. Although some aggressive clinical cases reported were patients that had undergone reconstructive surgery following breast cancer, suggesting predisposition to malignancy, many cases, including our patient, have no history of breast malignancy. Micrometastatic lymphoma disease may be present but undetected at initial diagnosis. Perhaps capsulectomy is not a sufficiently aggressive treatment for BIA-ALCL, as it would not eliminate micrometastases [16]; however, current expert opinion firmly disagrees with an obligatory chemo-/radiotherapeutic treatment of all patients with BIA-ALCL [9].

We would like hereby to direct attention to the importance of the recognition and cytological study of peri-prosthetic effusions, so as to enable early detection of a lymphomatoid proliferation and subsequent appropriate therapeutic management, because non-specific implant-related complications may result in delayed diagnosis [17]. Removed implants should always be examined. In addition, clinicians and pathologists should include this entity in the differential diagnosis of a lesion appearing in patients with breast implants. Although most cases have an indolent clinical course [9], the variety of presentations defined as "seroma" vs. "capsular involvement" emphasizes the importance of investigating a definitive method of diagnosis, management, and treatment of this disease [18]. The potential of effusion-only BIA-ALCL cases, a group for which this one may be a possible candidate, for latent metastatic dissemination may be underestimated. Clinicians and surgeons should be aware of the pitfalls of this neoplasm and keep complete medical history and close follow-up of female patients in such a setting. Integration of clinical and pathological data and awareness of these entities is crucial to prevent misdiagnosis and subsequent inappropriate therapy. On the other hand, a history of implants or chronic inflammation should be searched in the presence of an $\mathrm{ALK}^{-}$ALCL. The definitive role of adjunctive treatment forms, such as chemotherapy, immunotherapy, radiation, and HSCT for advanced disease, is still under investigation [19]. Our patient seems to have benefited so far from the combined treatment offered. 


\section{Compliance with ethical standards}

Conflicts of interest The authors declare that they have no conflict of interest.

\section{References}

1. Keech JA Jr, Creech BJ (1997) Anaplastic T-cell lymphoma in proximity to a saline-filled breast implant. Plast Reconstr Surg 100(2):554-555

2. Brody GS, Deapen D, Taylor CR, Pinter-Brown L, House-Lightner SR, Andersen JS, Carlson G, Lechner MG, Epstein AL (2015) Anaplastic large cell lymphoma occurring in women with breast implants: analysis of 173 cases. Plast Reconstr Surg 135(3):695705. doi:10.1097/PRS.0000000000001033

3. Miranda RN, Aladily TN, Prince HM et al (2014) Breast implantassociated anaplastic large-cell lymphoma: long-term follow-up of 60 patients. J Clin Oncol 32(2):114-120

4. Ye X, Shokrollahi K, Rozen WM, Conyers R, Wright P, Kenner L, Turner SD, Whitaker IS (2014) Anaplastic large cell lymphoma (ALCL) and breast implants: breaking down the evidence. Mutat Res Rev Mutat Res 762:123-132. doi:10.1016/j.mrrev.2014.08.002

5. Ganapathi KA, Pittaluga S, Odejide OO, Freedman AS, Jaffe ES (2014) Early lymphoid lesions: conceptual, diagnostic and clinical challenges. Haematologica 99(9):1421-1432

6. $\mathrm{Hu}$ H, Johani K, Almatroudi A, Vickery K, Van Natta B, Kadin ME, Brody G, Clemens M, Cheah CY, Lade S, Joshi P, Prince HM, Deva AK (2016) Bacterial biofilm infection detected in breast implant associated anaplastic large cell lymphoma. Plast Reconstr Surg 137(6):1659-1669. doi:10.1097/PRS.0000000000002010

7. Xu J, Wei S (2014) Breast implant-associated anaplastic large cell lymphoma: review of a distinct clinicopathologic entity. Arch Pathol Lab Med 138(6):842-846

8. Story SK, Schowalter MK, Geskin LJ (2013) Breast implantassociated ALCL: a unique entity in the Spectrum of CD30+ lymphoproliferative disorders. Oncologist 18:301-307

9. Kim B, Predmore ZS, Mattke S, van Busum K, Gidengil CA (2015) Breast implant-associated anaplastic large cell lymphoma: updated results from a structured expert consultation process. Plast Reconstr Surg Glob Open 3(1):e296. doi:10.1097/GOX.0000000000000268

10. Taylor KO, Webster HR, Prince HM (2012) Anaplastic large cell lymphoma and breast implants: five Australian cases. Plast Reconstr Surg 129:610e-617e
11. Adrada BE, Miranda RN, Rauch GM et al (2014) Breast implantassociated anaplastic large cell lymphoma: sensitivity, specificity, and findings of imaging studies in 44 patients. Breast Cancer Res Treat 147(1):1-14

12. Thompson PA, Lade S, Webster H, Ryan G, Prince HM (2010) Effusion-associated anaplastic large cell lymphoma of the breast: time for it to be defined as a distinct clinico-pathological entity. Haematologica 95(11):1977-1979

13. Swerdlow SH, Campo E, Pileri SA, Harris NL, Stein H, Siebert R, Advani R, Ghielmini M, Salles GA, Zelenetz AD, Jaffe ES (2016) The 2016 revision of the World Health Organization classification of lymphoid neoplasms. Blood 127(20):2375-2390

14. Laurent C, Delas A, Gaulard P, Haioun C, Moreau A, Xerri L, Traverse-Glehen A, Rousset T, Quintin-Roue I, Petrella T, Emile JF, Amara N, Rochaix P, Chenard-Neu MP, Tasei AM, Menet E, Chomarat H, Costes V, Andrac-Meyer L, Michiels JF, ChassagneClement C, de Leval L, Brousset P, Delsol G, Lamant L (2016) Breast implant associated anaplastic large cell lymphoma: two distinct clinicopathological variants with different outcomes. Ann Oncol 27(2):306-314

15. Clemens MW, Medeiros LJ, Butler HKK, Fanale MA, Horwitz S, Weisenburger DD, Liu J, Morgan EA, Kanagal-Shamanna R, Parkash V, Ning J, Sohani AR, Ferry JA, Mehta-Shah N, Dogan A, Liu H, Thormann N, Di Napoli A, Lade S, Piccolini J, Reyes R, Williams T, McCarthy CM, Hanson SE, Nastoupil LJ, Gaur R, Oki Y, Young KH, Miranda RN (2016) Complete surgical excision is essential for the management of patients with breast implantassociated anaplastic large-cell lymphoma. J Clin Oncol 34(2): $160-168$

16. George EV, Pharm J, Houston C, Al-Quran S, Brian G, Dong H, Hai W, Reeves W, Yang LJ (2013) Breast implant-associated ALKnegative anaplastic large cell lymphoma: a case report and discussion of possible pathogenesis. Int J Clin Exp Pathol 6(8):16311642

17. Jarjis RD, Hansen LB, Matzen SH (2015) The non-specific symptoms of breast implant-associated anaplastic large cell lymphoma resulting in delayed diagnosis: a case-based review. JPRAS Open 6: $1-4$

18. Hart AM, Lechowicz MJ, Peters KK, Holden J, Carlson GW (2014) Breast implant-associated anaplastic large cell lymphoma: report of 2 cases and review of the literature. Aesthet Surg J 34(6):884-894

19. Clemens MW, Miranda RN (2015) Coming of age: breast implantassociated anaplastic large cell lymphoma after 18 years of investigation. Clin Plast Surg 42(4):605-613. doi:10.1016/j.cps.2015.06. 006 